\title{
Metastatic calcification as a result of extensive bone metastases in a paediatric patient with parameningeal embryonal rhabdomyosarcoma
}

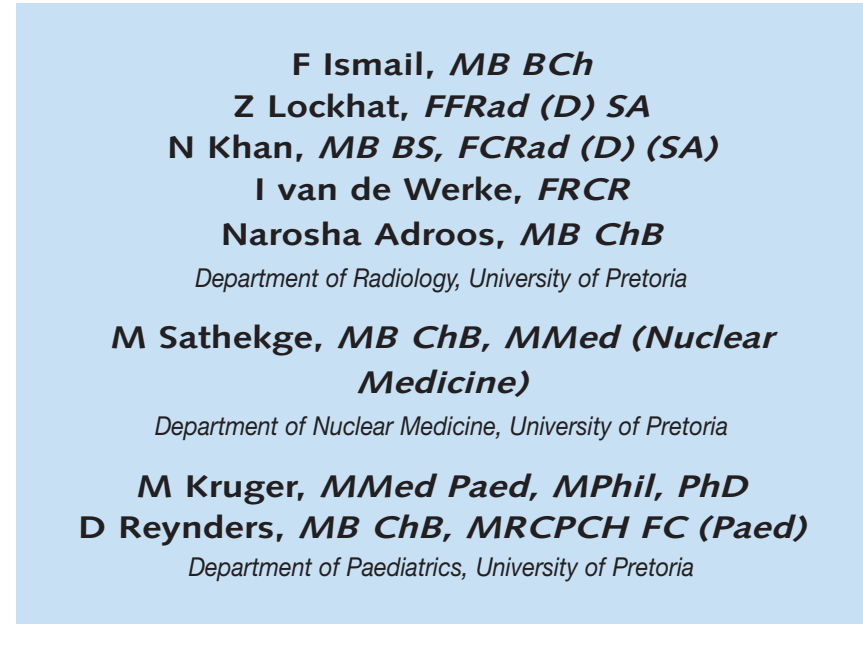

\section{Abstract}

We present a case of parameningeal embryonal rhabdomyosarcoma with the primary lesion arising in the middle and inner ear, with associated diffuse skeletal metastases and metastatic calcifications.

\section{Introduction}

Rhabdomyosarcoma is the third most common extracranial solid malignancy in children. ${ }^{1}$ Parameningeal rhabdomyosarcoma arises in sites adjacent to the meninges. Severe visceral metastatic calcification in this patient as a result of extensive bone metastases is described.

\section{Case presentation}

A 5-year-old boy presented with a chronic non-productive cough and right hip pain. During the clinical work-up, he was found to have a left pre-auricular mass. His biochemistry test results showed corrected cal-
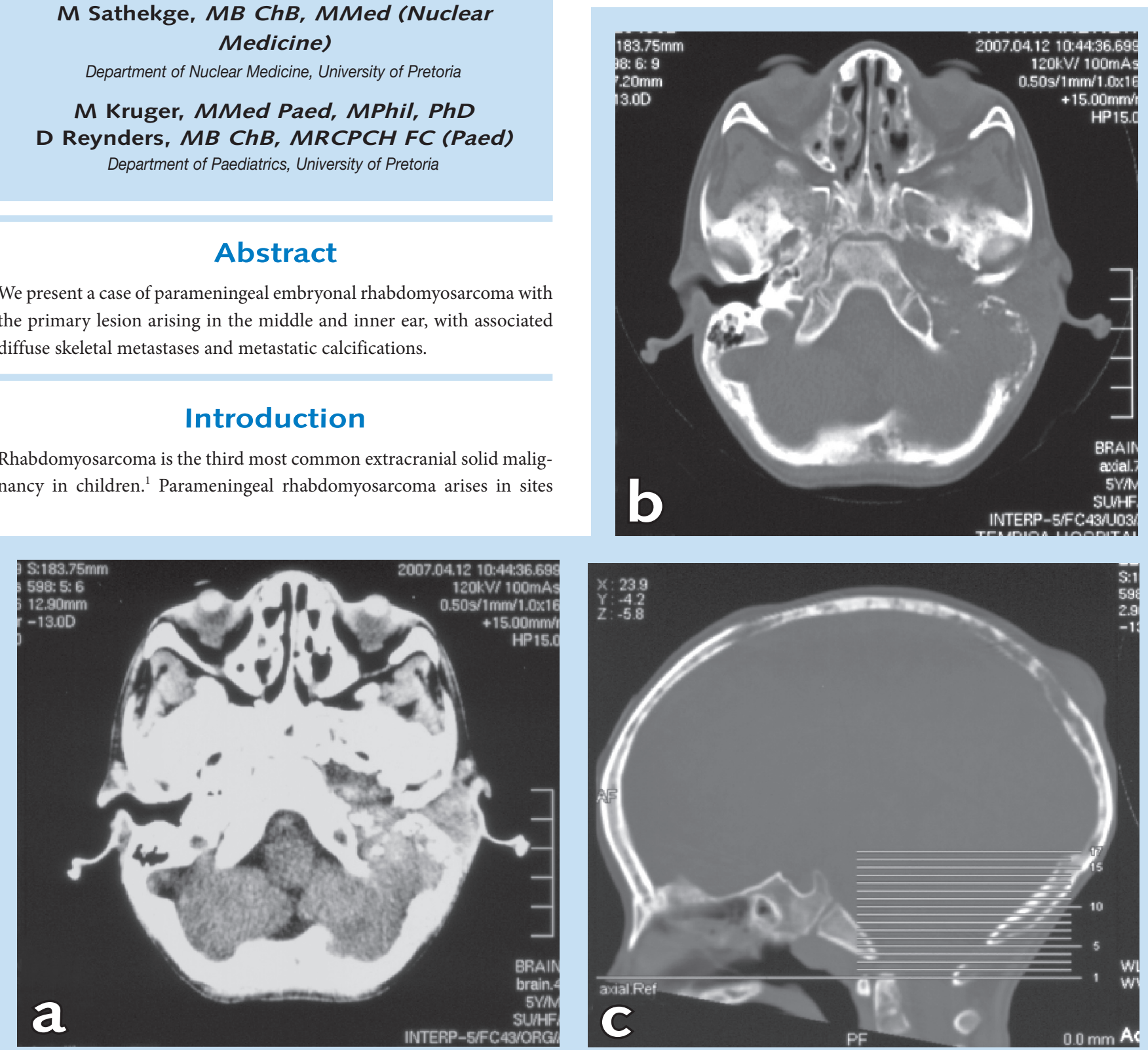

Figs 1a, 1b and 1c. Axial computed tomography in soft tissue (a), in bony windows (b) and sagittal view (c) demonstrates the left temporal mass eroding the temporal bone, extending anterior to the pre-auricular region. There are lytic lesions involving the inner and outer table of the calvarium and a soft-tissue metastatic mass in the occiput. 


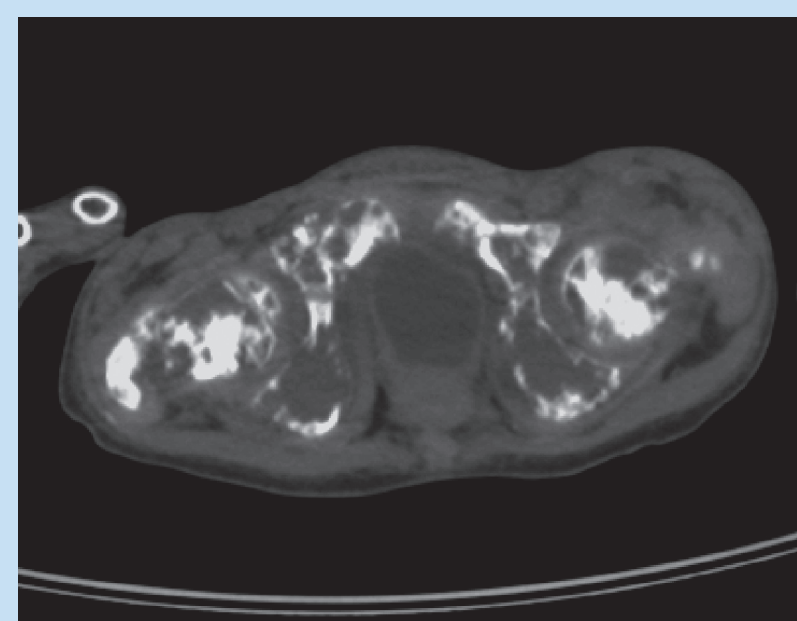

Fig. 2. Axial computed tomography scan in bone window at various levels demonstrating extensive lytic bone metastases.

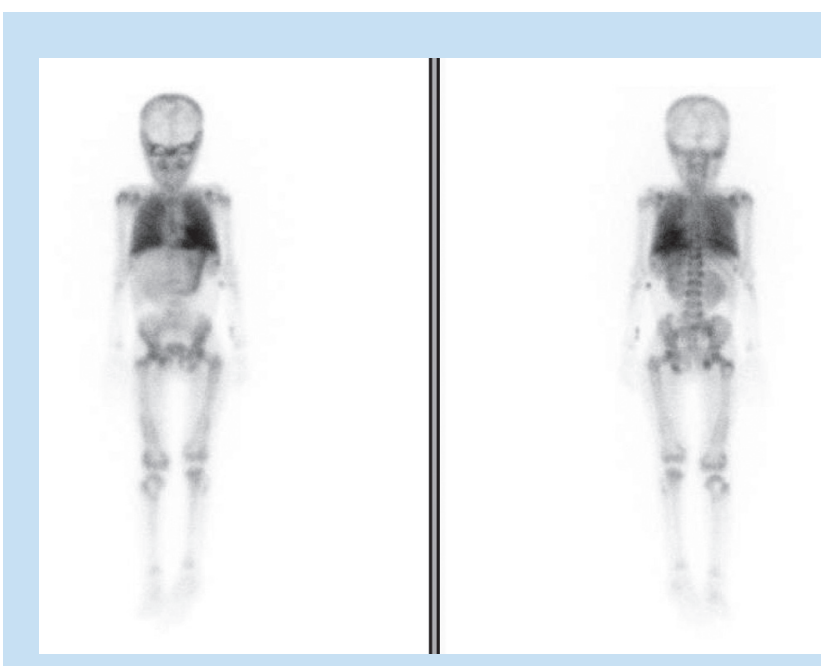

Fig. 3a. Tc-99m MDP whole body scan demonstrating diffuse visceral calcification in the lungs, myocardium and stomach, with no well-defined osteoblastic metastases noted.

cium of $4.19 \mathrm{mmol} / \mathrm{l}$ and phosphate of $1.73 \mathrm{mmol} / \mathrm{l}$, which were elevated in comparison with normal reference ranges.

Computed tomography (CT) (Figs 1a, b and c) of the brain showed a large, destructive lesion in the left ear cavity, extending anterior to the pre-auricular region and posterior to the mastoid air cells. There was destruction of the left temporal bone and external ear canal. There were lytic lesions in the calvarium and a soft-tissue mass in the occiput. The radiological diagnosis considered at this stage was metastatic neuroblastoma. CT of the chest, abdomen and pelvis was suggested to locate the primary lesion. The scan demonstrated diffuse lytic bony metastases (Fig. 2), involving all visible bones. The adrenal glands and para-aortic regions were normal, as were plain radiographs and CT of the lungs.

A Tc-99m-methylene diphosphonate (MDP) bone scan revealed diffuse metastatic visceral calcifications on the lungs, myocardium and stomach (Figs $3 \mathrm{a}$ and $\mathrm{b}$ ). These calcifications were not found on any of the other modalities.

Wedge biopsy of the pre-auricular lesion revealed a parameningeal

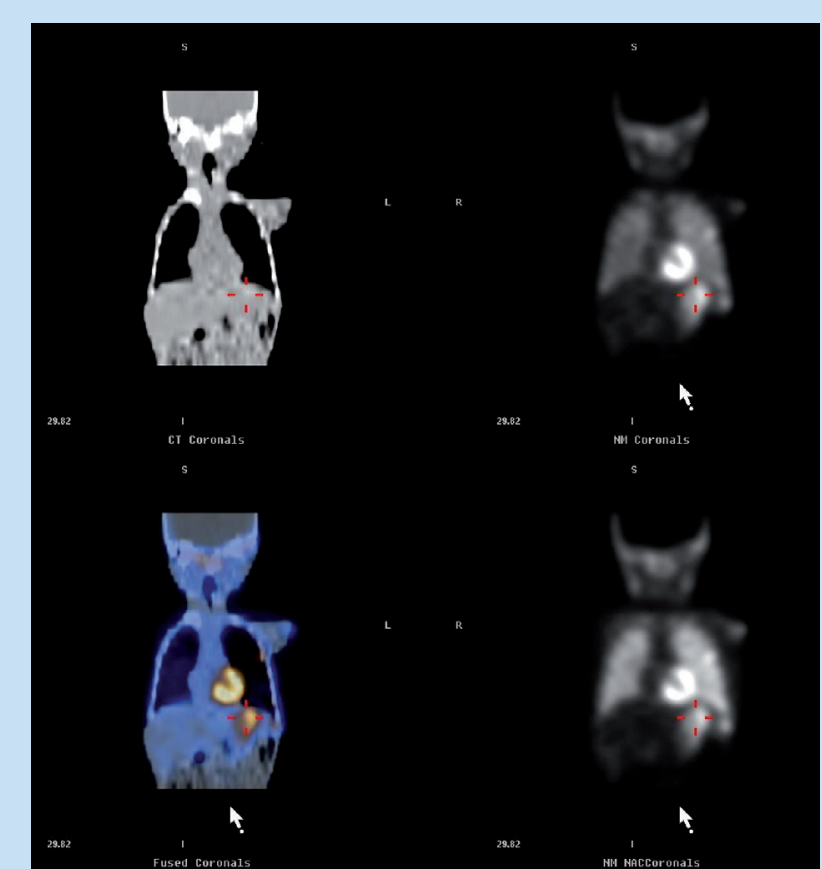

Fig. 3b. Tc-99m MDP SPECT-CT clearly localising the metastatic calcification in the lungs, myocardium and stomach

embryonal rhabdomyosarcoma. The primary tumour mass was in the left middle and inner ear. The patient presented at a late stage with diffuse bone metastases. He died a few days after the CT scan.

\section{Discussion}

Rhabdomyosarcoma is the third most common extracranial solid malignancy in children. It is a tumour of primitive mesenchymal cells committed to skeletal muscle differentiation which can occur in a variety of organs. There are three main histological types, viz. embryonal, alveolar and botryoid. ${ }^{1}$

'Parameningeal' sites are those primary sites which are anatomically adjacent to the meninges. These sites include the middle ear, mastoid, ear canal, oral cavity, paranasal sinuses and infratemporal fossa. ${ }^{1,2}$

Tumours originating in the middle ear and mastoid have a particularly bad prognosis. They are generally considered to be aggressive as they are in close proximity to vital structures, with a tendency to spread intracranially and the potential for meningeal involvement. ${ }^{2}$

Primary lesions can lead to bone remodelling and destruction, ${ }^{2}$ as was the case with our patient. At presentation, $42 \%$ of cases have cervical adenopathy and 58\% have distant metastases. ${ }^{3}$

Metastatic calcification usually results from a deposition of calcified products in otherwise normal tissues as a result of hyperphosphataemia with or without hypercalcaemia. It may affect mainly the lungs, blood vessels, stomach, periarticular soft-tissue, kidneys and myocardium, and to a lesser extent skin and skeletal muscles of extremities. ${ }^{4}$

Metastatic calcification affecting the myocardium and lungs is potentially lethal and is rarely detected before death, because of the absence of specific radiographic abnormalities. ${ }^{5}$ This lethal presentation was noted in our patient, as detected by Tc-99m MDP bone scan, and indeed the patient died soon after diagnosis. 
Metastatic calcification may occur in patients with chronic renal failure, primary hyperparathyroidism, extensive bone malignancy, hypervitaminosis $\mathrm{D}$, diffuse myelomatosis, and milk-alkali syndrome. ${ }^{4}$ Metastatic calcification may occur in the presence or absence of hypercalcaemia and is therefore not a prerequisite. At autopsy, metastatic calcification is reported to be present in up to $60-80 \%$ of patients with chronic renal failure. ${ }^{6}$ However, calcification is rarely identified on conventional chest radiographs.

McKay et al. recommend evaluation for bone metastases with Tc99m MDP bone scan or plain skeletal radiographs, and measuring the serum levels of parathyroid hormone $(\mathrm{PTH})$ and $\mathrm{PTH}$-related peptide, as well as the levels of $25-(\mathrm{OH})$ vitamin $\mathrm{D}$ and $1,25-(\mathrm{OH})$ vitamin $\mathrm{D}$ as this will point to the common causes for hypercalcaemia of malignancy and help to rule out non-malignant causes of hypercalcaemia. ${ }^{7}$

The lung is one of the primary sites of metastatic calcification deposition and, although most patients are asymptomatic, respiratory failure may develop. The deposition of calcium may be diffuse, predominantly lobar, or predominantly apical. The choice of imaging techniques is important in making the diagnosis. A chest radiograph may not be effective in the detection of metastatic calcification. ${ }^{6}$

CT - in particular, high-resolution CT - may or may not detect metastatic calcification. When pulmonary abnormalities are present, they most commonly consist of parenchymal opacification or poorly defined infiltrates that may simulate pneumonia or pulmonary oedema. On CT scans, multiple pulmonary nodules with or without calcification and calcifications of the vessels of the chest wall may be seen, more frequently on high-resolution chest CT. Other causes of pulmonary calcification include prior infections (tuberculosis, fungal or varicella), silicosis, sarcoidosis, metastatic tumour, rheumatic mitral valve disease, alveolar microlithiasis and idiopathic pulmonary ossification. However, these latter conditions do not usually display calcification of vessels of the chest wall. ${ }^{6}$ Furthermore, on CT there is relative lack of interlobular septal thickening, since the predominant sites of calcium deposition seen on pathological examination are the alveolar septa and, to a lesser extent, the pulmonary arterioles and bronchioles.

Detecting pulmonary uptake of bone-seeking radiopharmaceuticals is a valuable adjunct to diagnosis, and it has been suggested that bone scanning be used to screen for occult pulmonary calcifications. ${ }^{8}$ The scintigriphic pattern of dense uptake in the lung, frequently associated with stomach and kidney uptake in general, correlates with the distribution of calcification found at autopsy. ${ }^{8}$

In conclusion, the case discussed revealed a parameningeal embryonal rhabdomyosarcoma with diffuse skeletal metastases and visceral metastatic calcifications, and highlights the fact that chest radiographs and CT findings may be nonspecific and that the use of Tc-99m MDP scintigraphy is valuable to the diagnosis.

In patients with hypercalcaemia, it is important to exclude other benign causes for hypercalcaemia, which are more easily treatable as hypercalcaemia of malignancy may be resistant to treatment.

1. Wataru $\mathrm{W}$, Sutow MD, Lindberg MD, et al. Three year relapse-free survival rates in childhood rhabdomyosarcoma of the head and neck. Report from the Intergroup rhabdomyosarcoma study. Cancer 1982; 49: 2217-2221.

2. Greyling P, Andronikou S, Ackerman C, Schulze OC, Wieselthaler NA. Rhabdomyosarcoma - site matters S Afr J Radiol 2006; 10: 8-13.

3. Sutton D. A Textbook of Radiology and Imaging. 7th ed. Philadelphia: Elsevier Churchill Livingstone, 2003: 1529.

4. Centili A, Miron SD, Bellon EM. Nonosseous accumulation of bone-seeking radiopharmaceuticals. Radiographics 1990; 10: 871-881.

5. Casani F, Villanueva-Meyer J. Myocardial and lung uptake of Tc-99m-pyrophosphate using single photon emission computed tomography in a patient with end-stage renal disease and secondary hyperparathyroidism. Int Urol Nephrol 1996; 28: 569-574.

6. Hartman T, Muller N, Primack S, Johkoh T, Takeuchi N. Metastatic pulmonary calcification in patients with hypercalcemia: Findings on chest radiographs and CT scans. AJR 1994; 162: 799-802.

7. McKay C, Furman WL. Hypercalcemia complicating childhood malignancies. Cancer 2006; 72: 256-260.

8. Rosenthal D, Chandler H, Azizi F, Schneider P. Uptake of bone imaging agents by diffuse pulmonary metastatic calcification. $A J R$ 1977; 129: 871-874

9. Wharam MD. Rhabdomyosarcoma of parameningeal sites. Semin Radiat Oncol 1997; 7: 212-216.

10. Daya H, Chan HSL, Sirkin W, Forte V. Pediatric rhabdomyosarcoma of the head and neck. Is there a place for surgical management? Arch Otolaryngol Head Neck Surg 2000; 126: 468-472. 